\title{
Surface Roughness of Thin Gold Films and its Effects on the Proton Energy Loss Straggling
}

\author{
C. Celedón ${ }^{\dagger}$, M. Flores ${ }^{\dagger *}$, P. Häberle* ${ }^{*}$ and J. E. Valdés ${ }^{\dagger}$ \\ ${ }^{\dagger}$ Laboratorio Colisiones Atómicas, Departamento de Física, \\ Universidad Técnica Federico Santa María, Valparaíso, Chile \\ ${ }^{*}$ Laboratorio de SPM-UHV, Departamento de Física, Universidad Técnica Federico Santa María, Valparaíso, Chile \\ ${ }^{\ddagger}$ Instituto de Física, Pontificia Universidad Católica de Valparaíso, Valparaíso Chile
}

Received on 8 December, 2005

\begin{abstract}
We present a description of the effect of the surface roughness on the energy straggling associated to the energy loss distributions of protons transmitted through a self supported metallic thin foil. For this purpose we prepared a polycrystalline gold thin films using the standard sputtering method with different deposition rates. The statistics of the surface height distribution induced in these thin films were determined using Atomic Force Microscopy. The measured surface roughness allowed us to quantify the ion energy loss straggling in these samples for different deposition parameters and as a function of the incident ion energy.
\end{abstract}

Keywords: Ion Scattering; Atomic Force Microscopy; Self supported thin films

\section{INTRODUCTION}

At the present time there is a great interest to carry out stopping power cross section measurements of light ions at low energies in metallic targets using a transmission geometry [1]. For this purpose it is necessary to provide self supported thin films with a precise thickness and a known surface roughness. It is well known that the ion energy loss distribution has a Gaussian shape and the standard deviations of these distributions are directly related with the collision statistics, target characteristics (density, surface roughness, grain size) and experimental conditions such as energy resolution and beam angular divergence. These two last conditions are not very important in the energy straggling as will be shown below.

For stopping cross section measurements it is necessary to know the parameters that characterize the homogeneity of the target, such as: average thickness, roughness and grains size distribution of the film. In order to include the thickness of the layer, an indirect method, based on the measurement of the stopping power of the ion beams has been developed [2]. However, in order to establish statistical models of the surface roughness, it is necessary to make systematic measurements of these parameters and to correlate them with measurements of the energy loss straggling in the ion energy loss distributions. In this sense we need to characterize the roughness of the thin film by a direct and non destructive method.

In this report we focus in the study of the surface roughness of gold polycrystalline thin films and its effect in the energy loss straggling.

\section{EXPERIMENTAL DETAILS}

The gold thin films were grown by techniques of sputtering deposition at room temperature (RT) on a silicon substrate with the native oxide on the top layer. Roughness was characterized by Atomic Force Microscopy.

The $10 \times 5 \mathrm{~mm}^{2}$ Silicon substrates were cleaned in an ultrasonic bath with high purity methanol during 1 minute and the residual impurities cleaned with distilled water. The sub- strates were subsequently dried in an argon atmosphere before proceeding with the gold deposition.

The deposit of the gold thin films was carried out in a commercial sputtering system (SPI), with a quartz crystal balance (QCB). The sputtering system is evacuated using a small turbo molecular pump with an oil free rotary pump, reaching a pressure below 10 mTorr. Samples were prepared with three different deposition rates, $0.6 \AA / \mathrm{s}, 1.2 \AA / \mathrm{s}$ and $1.4 \AA / \mathrm{s}$, for a total nominal thickness of $130 \AA$. The purity of the films was monitored by Auger Electron Spectroscopy (AES) in the energy range between $0-1000 \mathrm{eV}$. Measurements of the surface roughness were obtained from topographic images from an Atomic Force Microscope (AFM, Omicron VT-AFM) in contact mode. For each case the measured values of roughness were correlated with the different deposition rates.

\section{RESULTS}

For each film we obtained a series of topographic images with different magnifications. Spatial representations of the surfaces are shown in Fig. 1. These images correspond to the $\mathrm{SiO}_{2}$ substrate, and a set of $130 \AA$ thick thin films grown with different deposition rates $(0.6 \AA / \mathrm{s}, 1.2 \AA / \mathrm{s}$ and $1.4 \AA / \mathrm{s}$, samples 1,2 and 3, respectively). Differences in the surface roughness are clearly observed between the clean substrate and the Au films. This particular substrate was chosen by its original low roughness. The RMS roughness of the $\mathrm{SiO}_{2} / \mathrm{Si}(111)$ shown in Fig. 1 is $0.51 \mathrm{~nm}$.

¿From the AES spectra of Fig. 2, we can appreciate the samples are pure gold thin films. The spectra of the different samples show the same number of peaks and each one of them is associated to a gold transition. We can also observe the films are free of oxygen and other surface contaminants. No signals associated to the substrate are detected; indicating the deposited film is uniformly grown over the substrate.

Topographic images were taken with the AFM in contact mode, set to take images, at different magnifications 

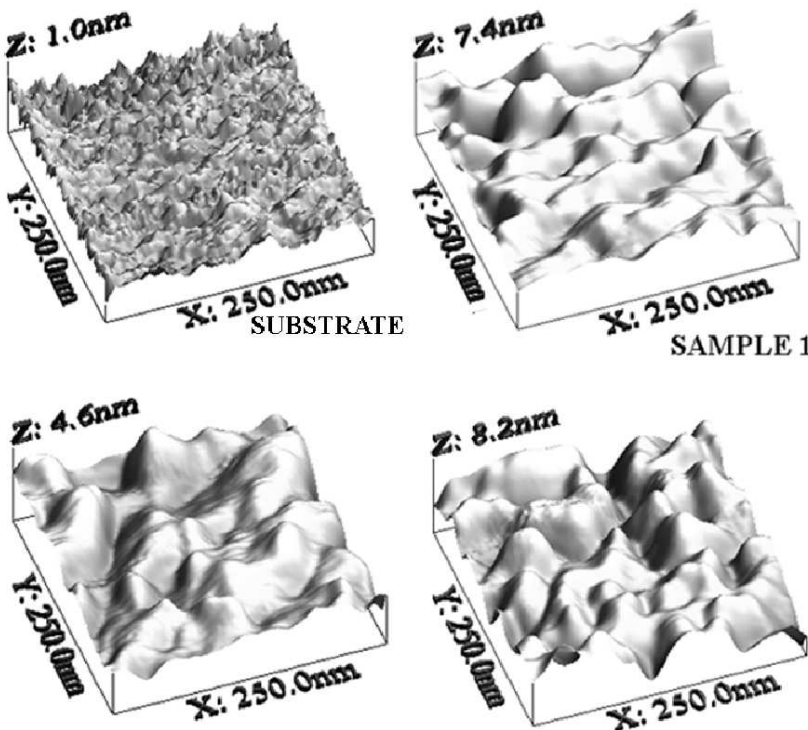

SAMPLE 2

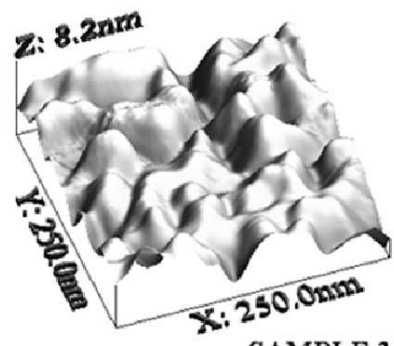

SAMPLE 3

FIG. 1: AFM Image of 130Å gold thin film, represented in a 256x256 pixels arrangement.

$\left(2500 \times 2500 \mathrm{~nm}^{2}, 1000 \times 1000 \mathrm{~nm}^{2}\right.$ and 250x250 $\left.\mathrm{nm}^{2}\right)$. Several images were taken over different sections of the sample. For topographic scans over small areas, the height distribution is narrower as expected. Finally the magnifications selected for the scans imply a separation of $1 \mathrm{~nm}$ between pixels. Measurements with higher magnifications did not contribute additional information regarding the surface roughness. The images used in this study have a magnification of $250 \times 250 \mathrm{~nm}^{2}$ with a normal force; $\mathrm{F}_{N}=5 \mathrm{nN}$, in contact mode.

The most important feature that we can observe from these images is a decoration of collapsed grains. These grains are distributed homogeneously with a pattern consistent with a polycrystalline film.

A quantitative analysis of the superficial roughness from these images is summarized in Table I. Here the normalized roughness $\rho$ of the films is presented as a function of the deposition rate ( $\bar{X}$ is the average width of the sample).

TABLE I: Surface roughness of Au thin films measured from AFM topographic images. The first column is the deposition rate. The second column is the normalized roughness. The third column shows the second moment of the height distribution.

\begin{tabular}{|l|l|l|l|}
\hline Sample & $\begin{array}{l}\text { Rate of } \\
\text { Deposition }(\AA / s)\end{array}$ & $\rho=\frac{\sigma_{X}}{\bar{X}}$ & $\mathrm{R}_{s k}$ \\
\hline Substrate & - & - & -0.21 \\
\hline 1 & 0.6 & 0.074 & 0.429 \\
\hline 2 & 1.2 & 0.044 & 0.019 \\
\hline 3 & 1.4 & 0.086 & 0.260 \\
\hline
\end{tabular}

The Amplitude Distribution Function (ADF, which is equivalent to the height distribution) is shown in Fig. 3. The

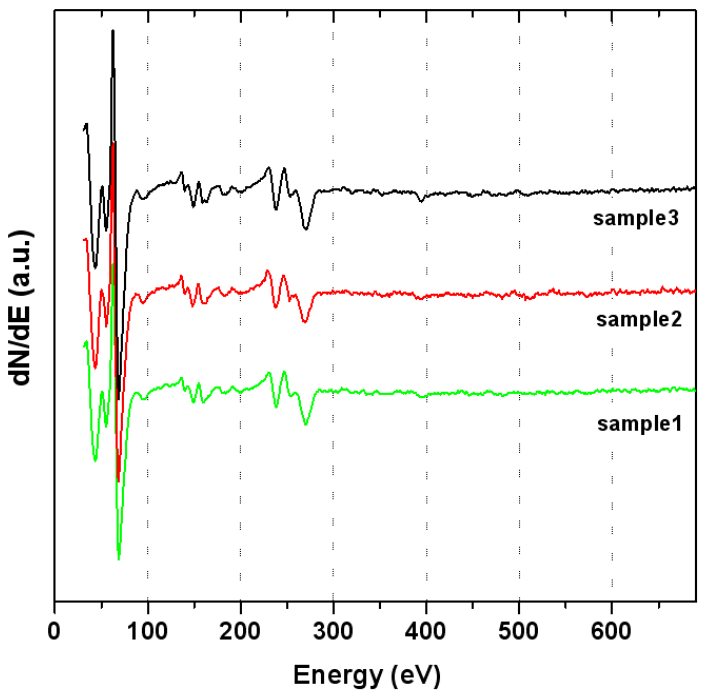

FIG. 2: AES for samples 1 to 3, at different deposition rates: $0.6 \AA /$ s, $1.09 \AA / \mathrm{s}$ and $1.2 \AA / \mathrm{s}$.

skewness values $\left(\mathrm{R}_{s k}\right)$ obtained from the ADF analysis indicates the values of the average roughness $\mathrm{R}_{a}$ for both substrate and samples. The negative sign of $\mathrm{R}_{s k}$ for the substrate indicates that the surface morphology is a rough surface with flat regions and a low density of valleys or dips.

Samples 1 and 3 exhibit a positive value of $\mathrm{R}_{s k}$, consistent with the presence of some high peaks out of an average flat plane, a phase, which is a characteristic of coalescence growth of nanoscale particles. The average grain size is about 25-30 $\mathrm{nm}$.

For sample 2 the value of $\mathrm{R}_{s k}$ is consistent with a more symmetrical topography, both valleys and peaks have almost the same weight and the ADF is close to a gaussian distribution. This is the type of thin films synthesized for technological applications.

\section{ENERGY LOSS STRAGGLING}

The information obtained from the surface roughness of the films allow us to model the effect of the surface topography over the energy loss distribution of ions transmitted through these thin films. In particular the effect on the energy loss straggling, i.e. the standard deviation of the loss distribution. The main parameters of the experimental distributions are the mean energy loss, energy loss straggling and its asymmetry. The origin of the asymmetry comes from two factors; the first one corresponds to the statistics of multiple scattering leading 


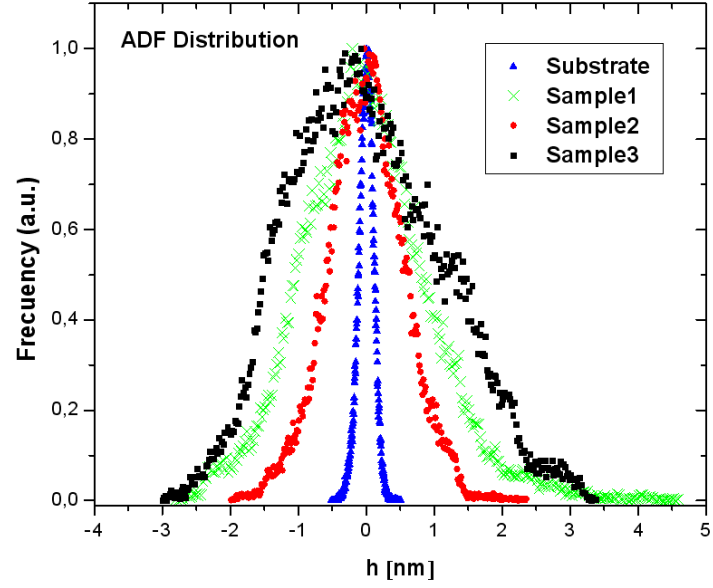

FIG. 3: ADF distributions of substrate and samples with a magnification of $250 \times 250 \mathrm{~nm}^{2}$.

to high energy losses. The second one is the different surface roughness distributions in each sample.

As is well known, both the energy loss and energy loss straggling are proportional to the incident ion velocity. These parameters can be written as a function of the energy as:

$$
\begin{gathered}
\frac{d E}{d x}=-k \cdot E^{\frac{1}{2}} \\
\Omega_{0}^{2}=<(\Delta E-<\Delta E>)^{2}>=\omega v^{2} \Delta x
\end{gathered}
$$

The constants $\mathrm{k}$ and $\mathrm{w}$ depend on the ion type and material with which the ion is interacting. These constants have been theoretically and experimentally determined. For the case of protons interacting with gold targets their values are [4]:

$$
k=0.047 e V^{1 / 2} \AA, \quad \omega=0.154 e V^{1 / 2} \AA^{-1 / 2}
$$

The experimental ion energy loss straggling has different contributions, such as, energy dispersion and angular divergence in the incident beam and energy resolution of the detection system. These parameters can enhance the width of the energy loss distribution. But they can be experimentally minimized in such a way they do not play an important role in the total energy straggling. For instance, normally the beam energy spread is less than $40 \mathrm{eV}$ at $5 \mathrm{keV}$ of the incident energy, and this implies around a $1 \%$ correction of the energy loss straggling. The beam divergence in the case of this type of transmission experiments is very small. The energy resolution is a correction factor in the final data. In addition to the statistics of collisions, there are two important factors that influence the energy straggling: multiple scattering effects and the surface roughness as previously described in Ref. [5]. The total energy loss straggling can be written as:
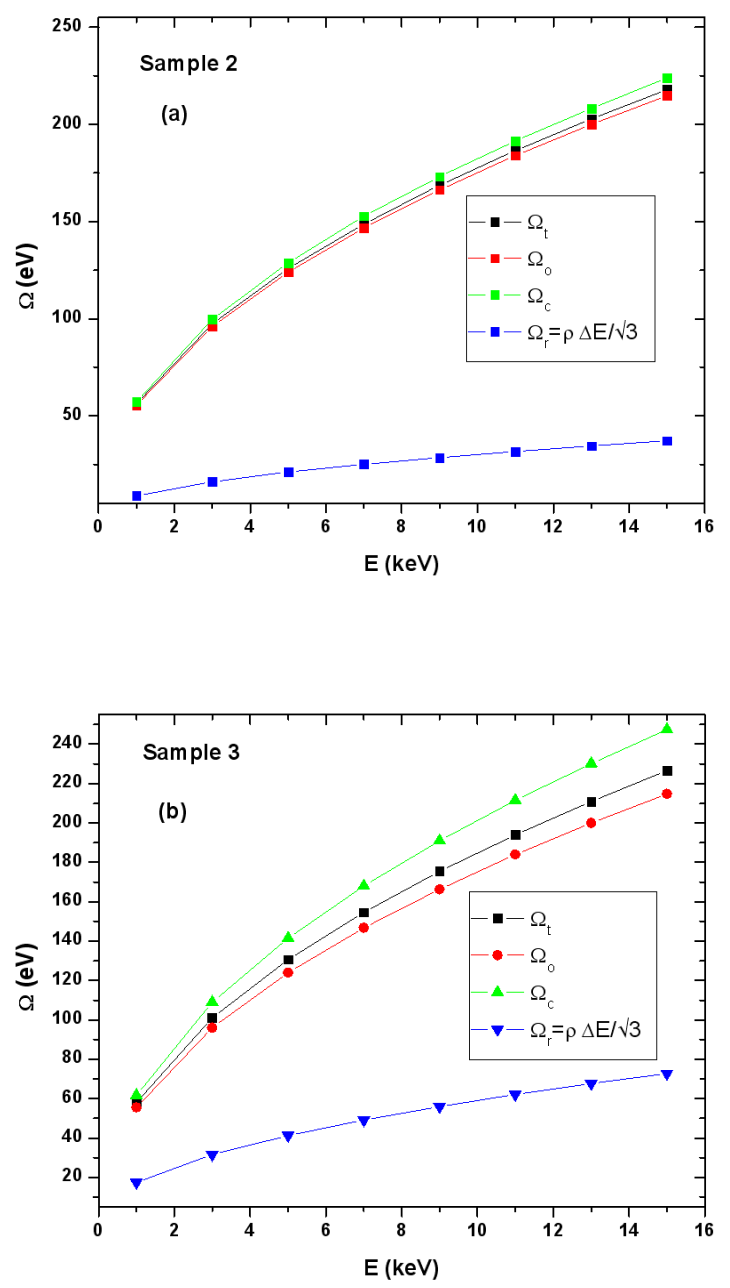

FIG. 4: Energy loss straggling for: (a) sample 2; and (b) sample 3.

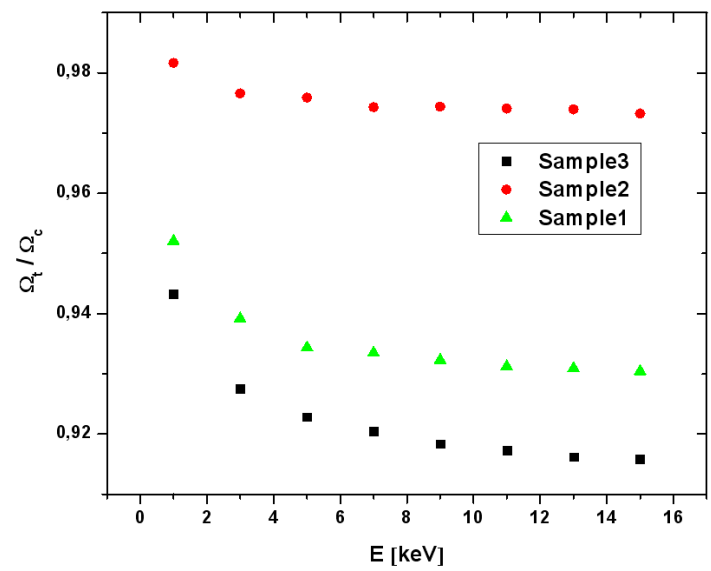

FIG. 5: Ratio of energy loss straggling, $\Omega_{t} / \Omega_{c}$ as a function of incident ion energy for the different samples. 


$$
\Omega_{c, t}^{2}=\Omega_{0}^{2}+\Omega_{r}^{2}
$$

Where, $\Omega_{0}$ is the "intrinsic" energy loss straggling due to the statistics of collision as shown in equation (2), $\Omega_{r}$ is the surface roughness contribution which can be expressed through a simple equation (see Ref. [3]). This relation for the straggling is given by: $\Omega_{r}^{2}=\rho^{2} \Delta E^{2}$ and can be derived from a roughness analysis of a film with only two steps. For a surface with an infinite number of steps the proper description is given in equation (4). This equation is a lower bound for the straggling parameter. Any experimental measurement of the straggling should be above this value.

$$
\Omega_{r}^{2}=\frac{\rho^{2} \cdot \Delta E^{2}}{3}
$$

In this equation $\rho$ is the roughness coefficient given by the standard deviation of the thickness distribution divided by the average thickness of the film, $\Delta \mathrm{E}$ is the mean energy loss of the energy loss distribution. Another way to obtain $\Omega_{r}$ is through the convolution of the thickness distribution $\boldsymbol{T}_{A D F}(\boldsymbol{x})$, obtained empirically with the AFM, and a theoretical energy loss distribution $\boldsymbol{G}\left(\boldsymbol{E}, \boldsymbol{E}_{0}, \boldsymbol{x}\right)$ that contains equations (1) and (2),

$$
F(E)=\int_{\min x}^{\max x} G\left(E, E_{0}, x\right) \cdot T_{A D F}(x) \cdot d x
$$

$\boldsymbol{F}(\boldsymbol{E})$ is the total energy loss distribution that contains all the information regarding the surface roughness and the collision statistics. We can compare both methods for obtaining the total energy loss straggling and verify which one is more appropriate to describe the effect of surface roughness on the experimental energy loss. First, we can obtain for this convoluted distribution the energy loss straggling $\left(\Omega_{t}\right)$ numerically, which corresponds to the standard deviation of $\mathrm{F}(\mathrm{E})$, and compare it with the energy loss straggling obtained from equations (3) and (4), $\Omega_{c}=\sqrt{\Omega_{0}^{2}+\Omega_{r}^{2}}$. In Fig. 4, we show the energy loss straggling as a function of the energy for two samples with minimum and maximum roughness coefficients. In each plot we represented the incident energy dependence of $\Omega_{t}, \Omega_{c}$, $\Omega_{o}$ and $\Omega_{r}$. From these graphs is clear the energy straggling is more significant for samples with high roughness coefficients. The ratio $\Omega_{t} / \Omega_{c}$ is shown in Fig. 5 as a function of the incident ion energy for all the samples considered in the analysis.

\section{SUMMARY}

We have described the surface roughness of thin gold films, deposited at room temperature with different depositions rates, through AFM analysis. The minimum surface roughness coefficient $(0.044 \mathrm{~nm})$ resulted from a deposition rate of $1.2 \AA / \mathrm{s}$. Under these conditions the surface exhibits three-dimensional growth, with a large number of peaks.

The new models proposed, based on the experimental surface roughness show deviations up to $8 \%$ in the value of the energy straggling (Fig.5) when compared to the standard model. Knowledge of the ADF allows the quantification of the surface roughness, which is an important input for understanding measurements of the energy loss straggling. The surface roughness in the self supported targets induces changes in the shape of energy loss distribution, mainly due to modifications of the total ion cross section over portions of the films with a different path length.

In order to check the results presented here, we will systematically determine the energy loss straggling at the Low Energy Ion Collisions Laboratory, at UTFSM. In this experiments we will use protons, incident on gold polycrystalline thin films.

\section{Acknowledgement}

We acknowledge support from the following grants: ICM N ${ }^{o}$ P02-054-F "Condensed Matter Physics"; Fondecyt $\mathrm{N}^{o} 1030175$; DGIP UTFSM N ${ }^{o} 11.04 .23$. M. Flores and C. Celedón acknowledge helpful input from P. Vargas.
[1] C. Archubi, C. Denton, J. E. Eckardt, G. H. Lantschner, F. Lovey, J. Valdés, C. Parra, F. Zappa, and N. R. Arista, Physica Status Solidi B 241, 2389 (2004).

[2] J.C. Eckardt and G.H. Lantschner, Thin Solid Films 249, 11 (1994).

[3] D. Kalz, G. Kreysh, and U. Muller Jahreis, Radiation Effects 36,
119 (1978).

[4] P. Vargas, J, E. Valdés, Phys. Rev. A 53, 1638(1996).

[5] M. M. Jakas and N. E. Capuj, Physical Review. A 40, 7369 (1989). 